\title{
The Effect of Surface Inclination on AFM Force-Curve Calibration and Evaluation
}

\author{
Judit Kámán $^{1)}$, Attila Bonyár1), and Róbert Huszánk ${ }^{2)}$ \\ ${ }^{1)}$ Department of Electronic Technology, Budapest University of Technology and Economic, Budapest, Hungary \\ ${ }^{2)}$ Institute for Nuclear Research, Hungarian Academy of Sciences, Debrecen, Hungary \\ kaman@ett.bme.hu
}

\begin{abstract}
The aim of the work is the investigation of the effect of surface inclination and tip-surface interactions on the calibration and evaluation of AFM (atomic force microscopy) force-curves. For this purpose both ideally flat and hard samples (Si wafer) and inclined but equally hard samples were used to measure the deflection inverse optical lever sensitivity, or InvOLS, of the cantilever. The inclined samples were polydimethylsiloxane (PDMS) irradiated and hardened by a proton beam to around 22 $G P a$, in a way, that the irradiated area forms a hill with inclination angles between 10-15. It is demonstrated that surface inclination can cause a $\pm 10 \%$ deviation in the obtained lever sensitivities, which in turn can cause even a cause a $-30 \%$ to $+50 \%$ relative error in the obtained elastic modulus for the surfaces, if neglected during force-curve evaluation..
\end{abstract}

\section{INTRODUCTION}

AFM (atomic force microscopy) is a widely used tool in several research areas to measure the topography, the electrical, the magnetic or the mechanical properties of nanometer-sized materials or structures. During AFM measurements a probe, which is positioned at the end of a cantilever is scanned over a sample, and the deflection of the cantilever is measured and used to calculate the interactions between the tip and sample. Most commercial AFMs use an optical method to detect the deflection of the cantilever. A laser beam reflects from the backside of the cantilever and returns into a position sensitive photodetector (PSPD), as in the illustration of Fig. 1, left. When the sharp tip mounted on the other side of the cantilever interacts with the sample, the cantilever bends and the laser-spot moves on the PSPD. So although the natural unit of the cantilever deflection would be metric, the measured signal in the PSPD is a voltage. The conversion factor is the so-called inverse optical lever sensitivity (InvOLS) in $[\mathrm{nm} / \mathrm{V}]$ whose determination is the first step at every AFM measurement. The precise knowledge of this factor is essential to get the correct value of many parameters, such as the high data, the exerted force by the tip, and the Young's modulus through the interaction force data, etc. The most widely used process to determine the
InvOLS is to perform measurements on ideally hard and flat samples, e.g. on silicon wafer or sapphire, where the conversion factor is determined as the slope of the resulting linear curve (Fig. 1, right) [1]. Since it can damage the tip several efforts have been made to replace it with non-destructive methods [2-4], but these methods requires special tools.

A serious problem with the classical calibration routine is that it presumes that only normal forces act on the cantilever. In the case of a realistic sample with inclined surface (or not completely normal indentation direction) the forces between the tip and surface could cause lateral bending or torsion in the cantilever, which means that the conversion factor determined during calibration will not be valid for these surfaces.

Therefore, the aim of this paper is to investigate this possible effect in detail, and determine the magnitude of the error which it can cause during calibration and also, during the subsequent calculation of Young's modulus, based on the obtained force-curves. For this purpose calibration curves measured on a flat $\mathrm{Si}$ wafer and a hardened PDMS (polydimethylsiloxane) with irregular surface will be used. 


\section{THEORY}

The determination of the exerted or measured force by AFM is typically calculated from the deflection $(D)$ of the cantilever using Hook's law (Eq. 1):

$$
F=k \cdot D \quad(1)
$$

where $k$ is the spring constant of the cantilever. This means that the precise knowledge of the $k$ and the $D$ is required. Several papers were published on the calibration of the spring constant; the most known methods are the thermal noise method [5], the Cleveland method [6] and the Sader method [7]. However, some of these methods already need the precise knowledge of the InvOLS. As it was mentioned, the widely used method the determination of the InvOLS is to do a contact-mode point-spectroscopy on a hard and flat surface. The tip is pushed against the surface and retracted meanwhile the deflection of the cantilever on the PSD in function of scanner motion is detected. If the surface is ideally hard, there is no indentation and the measured deflection-curve will characterize only the deformation of the cantilever. This linear relationship between the output of the PSPD in Volts and the sample position in nanometer is called the InvOLS $(\Delta z / \Delta V,[\mathrm{~nm} / \mathrm{V}])$, see Fig.1, right, and Eq. 2.

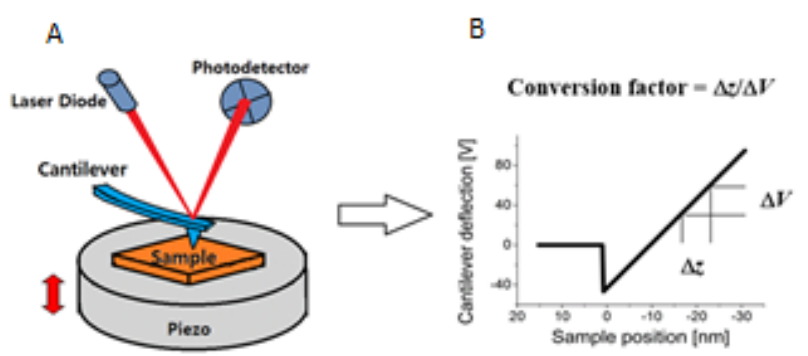

Fig. 1. Illustration of the calibration of the invers lever optical sensitivity (InvOLS), by performing a contact-mode point-spectroscopy (right). The slope of the linear part of the curve the conversion factor, or InvOLS.

$$
\Delta D=\frac{\Delta z}{\Delta V} \Delta V
$$

If the resulting deflection-curves are further scaled with the spring constant $(k)$ of the cantilever based on Hooke's law they are called force-curves.

For the evaluation of the deflection-curves a custom software was used, which fits the Hertz-Sneddon model [13] on the obtained contact-mode point-spectroscopy dataset to determine the Young's modulus $(E)$ as in Eq.
3 , where $z$ is the absolute piezo position, $z_{0}$ is the piezo position at the tip-sample contact point, $D$ is the relative deflection of the cantilever and $F_{\mathrm{a}}$ is the adhesion force.

$$
k D-F_{a}=\frac{2 E \tan (\alpha)}{\pi\left(1-v^{2}\right)}\left(z-z_{0}-D\right)^{2}
$$

Eq. 3 was used to calculate the Young's modulus of the tested, irradiated PDMS sample.

\section{METHODS}

\subsection{AFM measurements}

The measurements were performed with Veeco diInnova AFM microscope. SCM-PIC silicon-nitride tips were used to the contact-mode point-spectroscopy with $0.2 \mathrm{~N} / \mathrm{m}$ nominal spring constant. For the calculation of Young's modulus, the Poisson-ratio of the PDMS was assumed to be $v=0.5$, and 10 degree was used as the half opening angle of the tips $(\alpha)$.

\subsection{PDMS preparation}

The PDMS polymer samples were made using Sylgard 184 elastomer kit from Dow Corning [8]. The hydrosilylation reaction between the base polymer (vinyl-terminated poly(dimethylsiloxane), average $\mathrm{Mn}$ $\sim 125000$, viscosity $\sim 4500 \mathrm{cSt}$ ) and the curing agent (vulcanizer, contains platinum catalyst, dimethylmethyl-hydrogen-siloxane cross-linker and a tetramethyl-tetravinyl-cyclotretrasiloxane inhibitor) results in the cross-linking of the polymer chains to form the commonly used silicone elastomer. The silicone pre-polymer and the curing agent were thoroughly mixed in the recommended ratio of 10:1 and placed in ultrasonic bath for 5 minutes to remove the formed bubbles. Then the pre-polymer was poured in a Petri dish and cured for $20 \mathrm{~min}$ at a temperature of 100 ${ }^{\circ} \mathrm{C}$ and allowed to finish curing overnight at room temperature. After curing, the PDMS polymer substrate (about $2 \mathrm{~mm}$ thick) was cut to $2.5 \mathrm{~cm}$ diameter discs. The density of the PDMS samples prepared this way

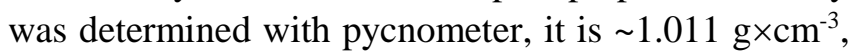
as it was measured in our earlier work [9]. The ion irradiations were done on these polymer disks. SRIM [10] calculations showed that the penetration depth for $2 \mathrm{MeV}$ protons is $\sim 81 \mu \mathrm{m}$ in the polymer.

\section{3 $\mathrm{H}+$ ion irradiation}

The energetic ions were produced by a $5 \mathrm{MV}$ single ended Van de Graaff accelerator at the facility in HASATOMKI, Debrecen, Hungary. The schematic view of 
the irradiation chamber can be seen in our previous work [11]. The target chamber is isolated allowing the measurement of absolute current and charge [12]. The irradiations were done in high vacuum environment. The beam size was $5 \mathrm{~mm}$ in diameter, and it was made homogeneous using a collimator system and a $0.51 \mu \mathrm{m}$ thin $\mathrm{Ni}$ foil. The energy of the protons bombarding the sample was $2 \mathrm{MeV}$, the beam current was about $\sim 15$ $n A$. The PDMS sample, which was used for this measurement was irradiated with $1.87 \times 10^{15}$ ions $/ \mathrm{cm}^{2}$ fluence.

\section{RESUlts AND DisCUSSION}

Although it is already known that the actual value of the InvOLS could depend on several parameters (such as the coating of the cantilever back-side, the optical settings, and the position of the laser spot), users usually choose the standard calibration method to be done at the beginning of the measurements on an ideally hard and flat surface. Here, we aim to investigate the surface inclination on the actual value of InvOLS. For this purpose we used a silicon wafer as an ideally flat and ideally hard surface (Young's modulus above $130 \mathrm{GPa}$ ) and an irradiated PDMS sample (fluence: $1.87 \times 10^{15}$ ions $/ \mathrm{cm}^{2}$, Young's modulus $\sim 22 \mathrm{GPa}$ ) which has an irregular, hill like surface topography, with contact angles between $10-15^{\circ}$, as can be seen in Fig. 3. This protrusion of the material was caused by the irradiation in the exposed $5 \mathrm{~mm}$ (diameter) area of the PDMS The exact same cantilever was used for the two experiments, its small spring constant $(0.2 \mathrm{~N} / \mathrm{m})$ ensures that both surfaces can be considered ideally hard and the measured deflection-curves will only characterize the response of the cantilever. As can be seen in Fig. 2, the deflection-curves obtained on the Si wafer have very small deviation (error below $0.5 \%$ ), but for the curves obtained on the PDMS sample (Fig. 3), this deviation is much higher (error around $10 \%$ ). Since the PDMS is also too hard to be deformed by such a soft tip we can rightly presume that the differences in the measured slope $(\Delta V / \Delta z$, which is the inverse of InvOLS) can only be attributed to local differences in the deformation of the cantilever caused by the varying incidence angles.

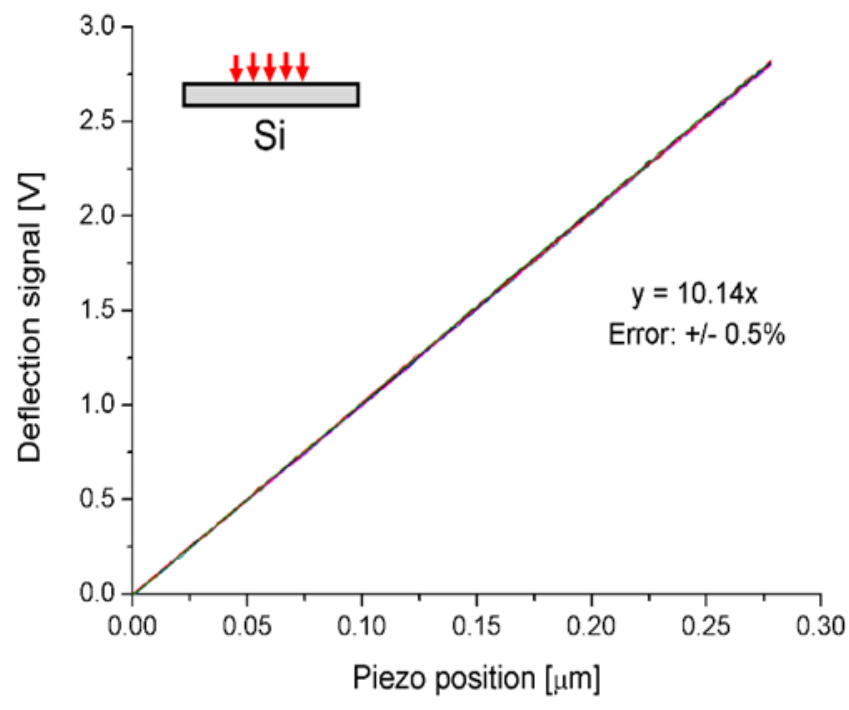

Fig. 2. : Calibration curves (deflection signal vs. piezo position) measured on a flat Si sample with a $0.2 \mathrm{~N} / \mathrm{m}$ AFM tip. The dashed line along with the equation represent the average of ten curves.

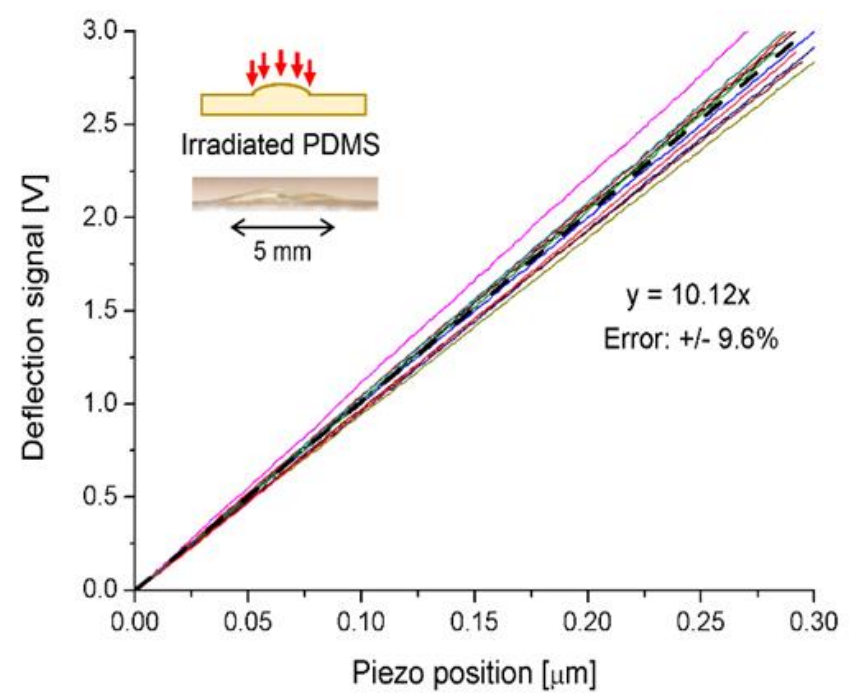

Fig. 3. Calibration curves (deflection signal vs. piezo position) measured on the irradiated, hard PDMS sample (fluence: $1.87 \times 1015$ ions $/ \mathrm{cm}^{2}$, Young's modulus $\sim 22 \mathrm{GPa}$ ) with a $0.2 \mathrm{~N} / \mathrm{m}$ AFM tip. The dashed line along with the equation represent the average of ten curves. Insert: the actual cross section of the PDMS sample, captured with an optical microscope.

It is now demonstrated, that if the calibration of the InvOLS was done prior to the actual measurements on an ideal sample, and if the surface of the sample has sufficient inclination the pre-determined InvOLS can contain an even $\pm 10 \%$ error compared to the actual one. If we would like to see how this error in the InvOLS contributes to the subsequent force-curve 
evaluation, and the calculation of the Young's modulus, we can use Eq. 3 to have an estimation. Based on this (by using the Hertz-Sneddon contact mechanics model), having a $\pm 10 \%$ error in the used InvOLS will in turn cause a $-30 \%$ to $+50 \%$ relative error in the determined Young's modulus of the measured sample.

\section{Conclusion}

It has been demonstrated, that surface irregularities (precisely inclinations in the $10-15^{\circ}$ range) can cause a significant, around $10 \%$ deviation in the determined InvOLS values, during calibration on hard samples. This means, that if the calibration of the cantilever is done prior to the actual measurement on an ideally hard and flat sample, the actual InvOLS, which is valid for the sample at hand, could be also significantly different. Based on the Hertz-Sneddon contact mechanics model, $10 \%$ error in the used and actually valid InvOLS could cause a $-30 \%$ to $+50 \%$ relative error in the determined Young's modulus of the measured sample. Currently we are working on a method which could yield InvOLS values during the measurement, for adaptive lever sensitivity calibration.

\section{ACKNOWLEDGEMENTS}

The technical assistance of the Van de Graaff accelerator operating staff at HAS ATOMKI is gratefully acknowledged. The research leading to these results has received funding from the ProProgressio foundation. Attila Bonyár and Róbert Huszánk are grateful for the support of the János Bolyai Research Scholarship of the Hungarian Academy of Sciences. This work was partially supported by the "ÚNKP" program of the Hungarian Government.

\section{REFERENCES}

[1] D. C. Lin, E. K. Dimitriadis, F. Horkay " Robust strategies for automated AFM force curve analysis--I.", J. Biomech. Eng., vol. 129, no. 3, pp 430-440, 2007.

[2] J. P. Cleveland, R. Proksch, M. J. Higgins, S. McEndoo, M. Polcik, S. P. Jarvis, and J. E. Sader,
"Non-invasive Determination Of Optical Lever Sensitivity in Atomic Force Microscopy", Rev Sci Instrum., Vol. 77, No. 1, Jan. 2006, pp. 013701013701.5

[3] C. J. Tourek, S. Sundararajan "Alternative method to determining optical lever sensitivity in atomic force microscopy without tip-sample contact", Rev Sci Instrum. Vol. 81, No. 7, Jul. 2010, pp. 073711073711.5

[4] N. P. D'Costa, J. H. Hoh, "Calibration of optical lever sensitivity for atomic force microscopy", Rev Sci Instrum. Vol. 66, No. 10, Oct. 1995, pp. 5096-5097

[5] Rui Li, Hongfei Ye, Weisheng Zhang, Guojun Ma, Yewang Su, An analytic model for accurate spring constant calibration of rectangular atomic force microscope cantilevers, Sci. Rep. 2015 Oct 29;5:15828. doi: $10.1038 /$ srep 15828 .

[6] J. P. Cleveland, S. Manne, D. Bocek, P. K. Hansma, A nondestructive method for determining the spring constant of cantilevers for scanning force microscopy, Review of Scientific Instruments 64, 403 (1993); https://doi.org/10.1063/1.1144209

[7] J. E. Sader, J. A. Sanelli, B, D. Adamson, J, P. Monty, Xingzhan Wei, S, A. Crawford,6 James, R. Friend, I, Marusic, P. Mulvaney, E. J. Bieske, Spring constant calibration of atomic force microscope cantilevers of arbitrary shape, REVIEW OF SCIENTIFIC INSTRUMENTS 83, 103705 (2012).

[8] DowCorning Corp. "Product information: Sylgard 184 Silicone elastomer" Form 10-898F-01.

[9] S.Z. Szilasi,; J. Kokavecz,; R. Huszank,; I. Rajta, Compaction of poly(dimethylsiloxane) (PDMS) due to proton beam irradiation, Applied Surface Science 257 (2011) 4612-4615.

[10] J.F. Ziegler, SRIM-2008.03. 〈http://www.srim.org>.

[11] R. Huszank, D. Szikra, A. Simon, S.Z. Szilasi, I.P. Nagy, 4He+ Ion Beam Irradiation Induced Modification of Poly(dimethylsiloxane). Characterization by Infrared Spectroscopy and Ion Beam Analytical Techniques, Langmuir 27 (2011) 3842.

[12] A. Angyal, Zs. Kertész, Z. Szikszai, Z. Szoboszlai, Study of Cl-containing urban aerosol particles by ion beam analytical methods, Nucl. Instrum. Methods. Phys. Res. B 268 (2010) 2211-2215.

[13] J. Domke,; M. Radmacher, Measuring the elastic properties of thin polymer films with the AFM, Langmuir, 14 (1998) 3320-3325. 